\title{
Innovation dans la prévention et la maîtrise de la rage dans le Nord : le projet pilote de gestion de la population de chiens dans la zone relevant de l'autorité sanitaire de la région de Weeneebayko
}

\author{
Lidstone-Jones $\mathrm{C}^{1 \star}$, Gagnon $\mathrm{R}^{1}$
}

\section{Résumé}

Contexte : Les communautés éloignées du Nord de l'Ontario sont confrontées à des problèmes uniques en ce qui concerne la prévention et la maîtrise de la rage. Avec de grandes populations de chiens en liberté qui présentent un risque élevé d'exposition à la rage par l'entremise de la faune et un manque d'accès régulier à des services vétérinaires et de vaccination, ces communautés présentent un risque plus élevé d'exposition à la rage que celles vivant dans les régions plus méridionales de la province.

Objectif : Offrir des données de base sur une nouvelle approche de maîtrise de la population de chiens dans la zone qui relève de l'autorité sanitaire de la région de Weeneebayko, dans le Nord de l'Ontario, laquelle approche est mise en œuvre dans le cadre d'un projet pilote durable, sans cruauté et rentable de gestion du nombre de chiens et d'amélioration de la sécurité publique.

Intervention : En 2015, l'autorité sanitaire de la région de Weeneebayko a lancé un vaste projet régional sur deux ans qui comprenait le micropiquage de tous les chiens de la région dans le but de quantifier et de surveiller les niveaux de population, la vaccination de ces animaux au moyen des vaccins de base habituels (y compris contre la rage) et l'essai pilote de l'utilisation d'un implant contraceptif injectable $\mathrm{GnRH}$ analogue agoniste chez les femelles. Les objectifs du projet comprenaient la maîtrise du nombre de chiens composant la population, la réduction des comportements agressifs chez les chiens de la communauté, la réduction du risque de contamination par la rage au sein des communautés, l'amélioration de la santé des chiens et l'éducation des membres de la communauté à propos de l'importance qu'il y a à maîtriser la population de chiens.

Résultats : En 2015, 513 chiens ont fait l'objet d'un micropiquage et ont été vaccinés dans le cadre du projet de l'autorité sanitaire de la région de Weeneebayko : 211 femelles et 301 mâles. Soit 76 chiennes en liberté ont reçu l'implant contraceptif, 113 chiennes ont été déterminées comme ayant déjà été stérilisées, et seulement 22 chiennes étaient soit trop jeunes, soit de trop petite taille (race de petit chien) pour recevoir un implant.

Conclusion : Tandis que les résultats finaux du projet ne sont pas encore disponibles, des résultats préliminaires, incluant les paramètres géographiques de la population de chiens et sa dynamique observée, appuient la faisabilité de poser des implants contraceptifs chez les femelles en tant qu'intervention primaire permettant de réduire rapidement et de façon rentable le nombre de chiens dans les régions éloignées du Nord et de réduire le risque de transmission de la rage.

\section{Affiliation}

${ }^{1}$ Autorité sanitaire de la région de Weeneebayko, Moose Factory (Ontario)

*Correspondance : caroline.lidstone-jones@ weeneebaykohealth.ca

Citation proposée : Lidstone-Jones C, Gagnon R. Innovation dans la prévention et la maîtrise de la rage dans le Nord : le projet pilote de gestion de la population de chiens dans la zone relevant de l'autorité sanitaire de la région de Weeneebayko. Relevé des maladies transmissibles au Canada 2016;42:145-50.

https://doi.org/10.14745/ccdr.v42i06a04f

\section{Introduction}

La nécessité d'améliorer les programmes de prévention et de maîtrise de la rage dans les communautés éloignées du Nord de l'Ontario est passée au premier plan au printemps 2013, lorsqu'un chiot de la réserve de la Première Nation Kashechewan a été diagnostiqué comme présentant la souche du virus de la rage du renard arctique, qui est endémique dans la région.

La Première Nation Kashechewan est une communauté accessible seulement par avion qui se trouve sur la côte de 
la baie James, en Ontario, et qui est desservie par l'autorité sanitaire de la région de Weeneebayko.

L'autorité sanitaire supervise les installations et les services médicaux de quatre communautés des régions côtières de la baie James et de la baie d'Hudson, en Ontario, y compris celles de Moosonee, Moose Factory, Fort Albany et Attawappiskat, et offre un soutien clinique aux postes infirmiers de Santé Canada de Kashechewan et Peawanuck. Avant l'incident du chiot, l'autorité sanitaire de la région de Weeneebayko avait établi la nécessité de résoudre le problème de la surpopulation des chiens et de réduire les risques de maladies zoonotiques comme une priorité dans son modèle intégré de prestation de services de santé publique.

En 2013, il a été extrêmement difficile d'effectuer une intervention de routine à la suite du diagnostic de la rage posé chez un chiot de la zone de Kashechewan. Cela était dû à un manque de ressources vétérinaires et de couverture vaccinale contre la rage, couplé avec l'absence de stratégies de gestion durable de la population de chiens. La situation a été encore davantage compliquée par le manque d'accès à de l'information fiable sur la taille de la population de chiens à risque. Parmi les autres enjeux figuraient l'identification fiable, le confinement et la surveillance des contacts entre le chiot infecté et les chiens de la zone de Kashechewan ainsi que les moyens à prendre pour que tous les autres chiens de la communauté soient vaccinés de manière à prévenir la propagation de la maladie.

Ces enjeux ont conduit un certain nombre de gouvernements clés et de partenaires de la santé animale à se réunir pour collaborer l'élaboration d'une solution régionale novatrice. Le projet pilote de gestion de la population de chiens de la zone relevant de l'autorité sanitaire de la région de Weeneebayko est un partenariat entre cette dernière, chacune des communautés de la région, le ministère de la Santé et des Soins de longue durée, Santé Canada, le Bureau de santé de Porcupine et Dogs With No Names, une organisation pionnière dans l'utilisation de méthodes différentes pour la contraception canine au Canada. La région couverte par l'autorité sanitaire de Weeneebayko s'étend sur la totalité de la côte ouest de la baie James et sur une partie de la côte de la baie d'Hudson jusqu'à Peawanuck (Winisk), des communautés étant réparties le long de la côte, tel que le montre la figure 1 ci-après, et la population humaine comptant environ 11860 personnes.

Lorsque nous avons planifié une intervention conçue pour traiter les enjeux particuliers auxquels sont confrontées les communautés éloignées du Nord, il nous a semblé évident que l'utilisation de moyens durables de maîtriser la population de chiens devait faire partie intégrante des programmes de prévention de la rage. En 2013, en s'appuyant sur leurs observations et grâce à la contribution de membres des communautés, des infirmières de Santé Canada dans chaque communauté ont produit des estimations initiales de la population totale de chiens, qui s'établissait à environ 1330 animaux dans la région de Weeneebayko.

Avant 2015, les six communautés éloignées visées par le projet de l'autorité sanitaire de la région de Weeneebayko avaient un accès variable aux programmes occasionnels de stérilisation chirurgicale des chiennes qui étaient offerts par différentes cliniques vétérinaires ou organisation se rendant sur place par
Figure 1 : Carte de la région côtière de la baie d'Hudson et de la baie James en Ontario, les communautés desservies par l'autorité sanitaire de la région de Weeneebayko étant indiquées par des cercles rouges

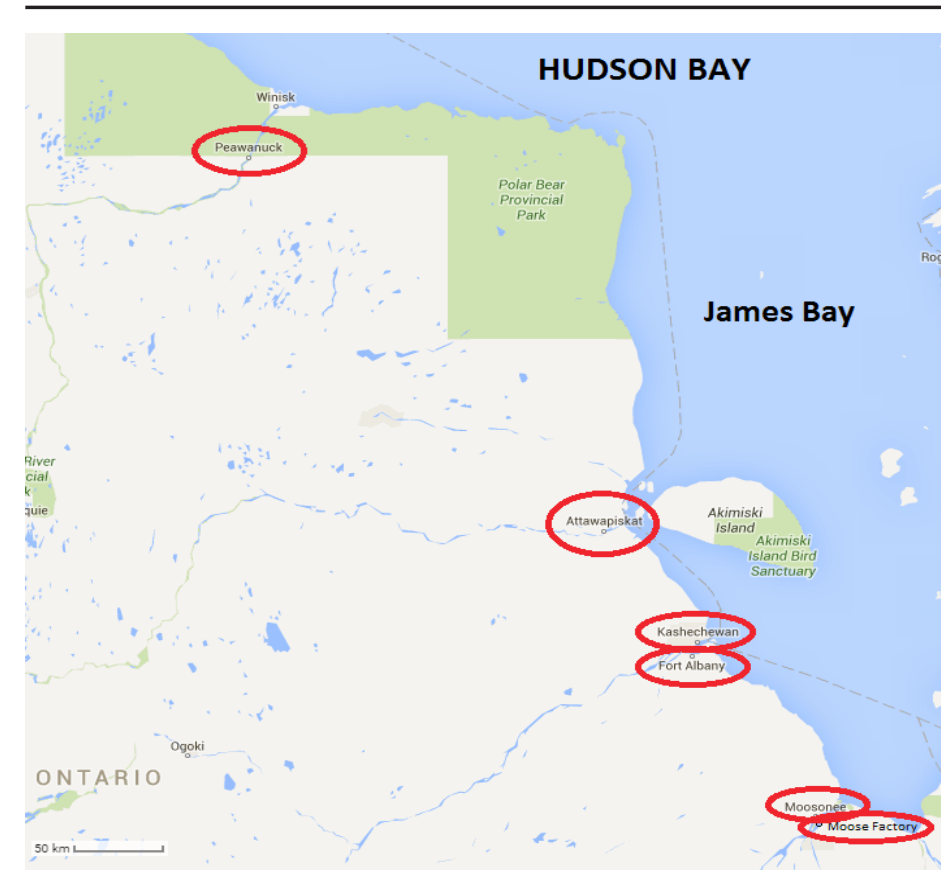

avion. Cependant, le manque d'approche régionale uniforme, de pair avec le peu (ou l'absence) de partage d'information entre les groupes ou les personnes offrant l'assistance vétérinaire à la région, les piètres taux de survie des chiens stérilisés chirurgicalement dans les communautés et les coûts très importants associés aux actes chirurgicaux dans la région se sont traduits par un succès limité de la maîtrise des populations de chiens en général.

La stérilisation chirurgicale des chiens en liberté dans cette région pose plusieurs problèmes importants, dont bon nombre sont insurmontables. Le transport de l'équipement chirurgical et d'anesthésie dans la région et au sein de la région est difficile sur le plan logistique et coûteux, et toutes les communautés ne disposent pas d'installations pour l'établissement d'une clinique de stérilisation chirurgicale efficace. Les communautés ne sont reliées entre elles que par des routes de glace qui sont ouvertes durant deux à trois mois pendant l'hiver, de sorte que les déplacements entre la plupart des communautés se font par les airs durant la plus grande partie de l'année. En outre, le climat nordique pose ses propres difficultés : les zones abdominales rasées et la récupération à la suite de l'utilisation d'agents anesthésiques durant les procédures de stérilisation se traduisent par de hauts taux de morbidité et de mortalité post-opératoires chez les chiens en liberté aux températures inférieures à zéro degré Celsius en hiver, et les animaux peuvent souffrir d'un inconfort élevé durant les mois d'été en raison des moustiques et d'autres insectes piqueurs.

Les expériences précédentes menées par Dogs With No Names avec d'autres communautés des Premières Nations de l'Alberta et du Labrador ont montré qu'il y avait habituellement un 
rapport de deux pour un chez les mâles et les femelles en liberté de ces communautés. Cela semble se produire pour

deux raisons : une diminution du taux de survie chez les femelles gestantes et allaitantes (dû aux exigences plus élevées en énergie pour la survie) et une discrimination active de la part des propriétaires ou des soigneurs de chiens à l'égard des femelles en raison de difficultés qu'il y a à composer avec les problèmes des portées et du cycle des chaleurs. L'espérance de vie moyenne des chiennes dans cet environnement est estimée à seulement trois ans (communication personnelle; Dre Judith Samson-French, docteur en médecine vétérinaire, 4 avril 2014).

En raison du nombre limité de chiennes en liberté disponibles, celles qui sont en chaleur tendent à créer un certain chaos au sein de la communauté, des meutes de mâles essayant de se reproduire avec elles tous en même temps. Cela engendre de l'agressivité entre les chiens, laquelle peut être redirigée vers des humains, posant un grave problème de sécurité au sein des communautés.

En conséquence, la solution la plus efficace et rentable à la surpopulation et aux comportements de meute agressifs associés aux chiens en liberté dans les communautés nordiques est de prévenir le cycle des chaleurs chez les chiennes par la stérilisation (soit de manière chirurgicale, soit en utilisant des implants contraceptifs). Cela permet de prévenir les comportements de meute problématiques et la reproduction. Les chiens mâles peuvent aussi être stérilisés pour en réduire le nombre au sein de la population, mais il est plus pratique et efficace de cibler les femelles. En outre, une étude récente examinant l'effet de la stérilisation chirurgicale et de la stérilisation chimique sur les comportements des mâles en liberté au Chili a montré que, tandis que la castration chirurgicale n'entraînait aucune réduction de l'agressivité ou de l'activité sexuelle, la stérilisation chimique menait en réalité à une élévation de l'agressivité des mâles à l'égard des autres chiens et ne se traduisait par aucun changement dans l'activité sexuelle (1).

Un certain nombre d'auteurs ont examiné la mesure et la maîtrise des populations de chiens dans des régions où la rage canine est endémique, comme en Inde et aux Philippines (2,3). Malgré des différences environnementales importantes susceptibles d'avoir une incidence sur les paramètres démographiques de la population canine dans les régions nordiques (p. ex. le climat nordique accompagné d'hivers rigoureux), une leçon importante que l'on peut tirer d'études antérieures menées dans d'autres parties du monde est l'importance qu'il y a à comprendre la dynamique de la population de chiens dans un milieu particulier (y compris l'espérance de vie à la naissance et les taux de mortalité au début de la vie) si l'on veut gérer de façon efficace les chiens en liberté (4). La compréhension de la dynamique de la population de chiens fait clairement partie intégrante de l'élaboration d'interventions dans les communautés éloignées du Nord et de l'évaluation de l'efficacité de celles-ci.

Cependant, nous disposons de peu d'information scientifique ou de lignes directrices publiées concernant les stratégies efficaces disponibles pour le contexte du Nord de l'Ontario, où de grandes populations de chiens en liberté ne sont pas elles-mêmes des espèces réservoirs pour le virus de la rage, mais posent un risque important parce qu'elles entrent régulièrement en contact avec des espèces fauniques réservoirs dans la région.
Après la tenue de vastes consultations et une étude soignée, le projet de l'autorité sanitaire de la région de Weeneebayko a été conçu autour de cinq objectifs :

1. Stabiliser sans cruauté les populations de chiens au sein et autour des communautés des Premières Nations à des niveaux durables et susceptibles d'être gérés;

2. Réduire l'agressivité chez les chiens et le risque de blessures qui en résulte parmi les membres de la communauté;

3. Réduire le risque de transmission de la rage et d'autres maladies depuis les chiens possédant un maître et les chiens en liberté vers des membres de la communauté;

4. Améliorer la santé globale des chiens de la communauté;

5. Éduquer les membres de la communauté quant à l'importance qu'il y a à maîtriser la population de chiens et aux incidences sur la santé publique pour toute la communauté.

Après le cycle initial des activités de terrain menées en 2015, cet article présente les résultats initiaux du projet de l'autorité sanitaire de la région de Weeneebayko concernant le nombre de base de chiens dans la population et l'information sur la démographie canine de base pour la région et représente la première information publiée sur les populations de chiens dans la région côtière de la baie d'Hudson et de la baie James, dans le Nord de l'Ontario.

\section{Intervention}

L'approche de régulation des populations choisie pour le projet de l'autorité sanitaire de la région de Weeneebayko consistait à utiliser 9,4 mg de Suprelorin ${ }^{\circledR}$ (acétate de desloréline), un implant contraceptif non chirurgical de la GnRH. Venu tout droit d'Australie dans le cadre du programme de distribution de médicaments d'urgence de Santé Canada, l'implant inhibe provisoirement le système reproductif endocrinien des femelles et empêche la production conjointe d'hormones hypophysaires et sexuelles pendant 12 à 18 mois. Les effets de l'implant sont semblables à ceux observés à la suite d'une stérilisation, mais s'inversent après la dissipation du contenu de desloréline de I'implant. Cependant, compte tenu de la courte durée de vie de la majorité des chiennes errantes dans le Nord, la durée d'efficacité de l'implant suffirait à stériliser une chienne pendant presque toute sa vie, surtout s'il est administré pendant deux années consécutives.

Étant donné que les communautés de l'autorité sanitaire de la région de Weeneebayko sont toutes éloignées, l'arrivée d'autres chiens provenant d'autres régions est limitée. Par conséquent, I'utilisation de contraceptifs injectables pendant deux années consécutives vise à stabiliser et à réduire les populations de chiens d'ici à la troisième année du programme à tel point que les interventions chirurgicales de stérilisation des chiennes plus âgées avec des taux de survie éprouvés sont plus efficaces en raison du nombre moins élevé de chiennes devant être opérées.

L'utilisation d'un implant contraceptif non chirurgical chez les chiennes présente des avantages uniques, notamment la rapidité, la manipulation minimale des chiennes, le potentiel d'utilisation durant toute l'année, le coût marginal et l'absence de complications postopératoires. Les implants de Suprelorin ${ }^{\circledR}$ 
utilisés dans le cadre du projet de l'autorité sanitaire de la région de Weeneebayko ont été fortement utilisés comme moyen de contraception dans de nombreux zoos et chez de nombreuses espèces sauvages, et se sont avérés efficaces chez les chiennes reproductives par le passé (5).

Au cours des semaines précédant l'arrivée de l'équipe sur le terrain du projet de l'autorité sanitaire de la région de Weeneebayko dans chaque collectivité en 2015, une campagne d'intervention communautaire et de sensibilisation du public a été lancée et avait recours au publipostage direct auprès des membres des communautés, aux affiches dans les communautés, aux messages sur Facebook et aux annonces publicitaires à la radio et à la télévision. Une approche en

deux phases a été adoptée pour pouvoir approcher les chiens : les membres de la communauté commençaient par apporter les chiens à une "station de traitement » centralisée, puis des approches mobiles de " porte-à-porte » et " rue-à-rue » ont été entreprises pour le reste des chiens dans la communauté; une équipe travaillait à partir d'un véhicule pour capturer les chiens errants. Une permission et un consentement éclairé écrits relatifs à la manipulation de chaque chien ont été obtenus auprès des habitants des maisons avoisinantes pour les chiens ayant un propriétaire et auprès du Chef et du Conseil pour les chiens errants sans propriétaire. L'efficacité des campagnes de sensibilisation du public d'une année à l'autre sera évaluée en comparant la proportion des résidents de la communauté qui acceptent d'apporter leurs chiens à la « station de traitement " centralisée au lieu de demander à l'équipe sur le terrain du projet de faire du porte-à-porte en 2016.

Chaque chien errant rencontré dans la communauté a été capturé en toute sécurité et a été nourri avec des aliments en conserve contenant un vermifuge. Un anesthésique local $\left(0,5 \mathrm{~mL}\right.$ de Carbocaine $\left.{ }^{\mathrm{MC}}\right)$ a été injecté dans le tissu sous-cutané entre les omoplates du chien, puis des vaccins contre la rage et la pneumonite féline, I'adénovirus-2, le parvovirus et la parainfluenza (DA2PP) ont été administrés dans les quartiers arrière du chien, une fois que l'anesthésique faisait son effet. Après la vaccination, une micropuce a été injectée entre les omoplates avec une aiguille de calibre 14. Si le chien était une femelle, un implant contraceptif de 9,4 mg de Suprelorin ${ }^{\circledR}$ était aussi injecté entre les omoplates de l'animal. La bonne insertion de la micropuce était confirmée par un lecteur de micropuce. La procédure complète, du début à la fin, après la capture du chien, ne prenait pas plus de cinq minutes par chien.

Tous les renseignements relatifs à chaque chien manipulé par l'équipe du projet étaient consignés dans un registre communautaire des chiens équipés de micropuces. Pour aider à bien repérer les chiens errants de la communauté déjà capturés dans le cadre du projet, tous les chiens vaccinés et munis de micropuces portaient une étiquette de vaccination antirabique très visible sur un collier, qui permettait également d'estimer le nombre de chiens dans chaque communauté qui n'avaient pas été capturés en 2015.

Pour l'année 2016, les opérations sur le terrain commenceront avec l'évaluation de chaque chien présenté ou capturé afin de déterminer s'il est muni d'une micropuce, pour ainsi définir son identité, son statut de vaccination et de reproduction. Chaque chien déjà capturé en 2015 fera l'objet d'un suivi en 2016 et sera vacciné une seconde fois contre la rage avec un vaccin antirabique de trois ans qui réduira de manière significative le risque de transmission de la rage dans la région. En 2016, tout nouveau chien et tous les chiens qui n'ont pas déjà été capturés par l'équipe sur le terrain en 2015, se verront également insérés une micropuce, seront vaccinés (dans le cas d'une femelle) et recevront un implant durant la deuxième année du projet.

La baisse du nombre de chiennes en rut (due à l'implant contraceptif) devrait diminuer le nombre de comportements agressifs de la meute chez les chiens mâles au sein des communautés. L'efficacité de cette stratégie visant à réduire l'agressivité et à améliorer la sécurité publique sera évaluée dans le cadre de rapports produits par les services policiers de la nation Nishnawbe-Aski pour chacune des communautés des Premières Nations en mentionnant le nombre d'appels liés aux chiens (p. ex. meutes agressives, attaques de chiens, combats de chiens, etc.) reçus par les services policiers de la nation Nishnawbe-Aski chaque année entre 2014 et 2017.

\section{Résultats}

Parmi les quelque 850 chiens identifiés dans la région en juin et en juillet 2015 par l'équipe sur le terrain, 513 (60\%) ont été capturés et vaccinés, et ont reçu une micropuce, créant ainsi un registre des chiens munis d'une micropuce pour chacune des communautés. Les résultats de l'intervention de 2015 pour l'ensemble de la région sont indiqués dans la figure 2 ci-dessous. Parmi les 513 chiens capturés, 211 (environ $40 \%$ ) étaient des femelles, tandis que 301 (environ 60 \%) étaient des mâles. Parmi les 211 femelles, $113(54 \%)$ avaient déjà été stérilisées et 98 (46\%) n'étaient pas stérilisées. Parmi les femelles non stérilisées, 76 (76 \%) ont reçu un implant contraceptif. Les 22 autres femelles non stérilisées étaient trop jeunes (âgées de moins de huit semaines) ou trop petites ( $p$. ex. les races naines vivant à l'intérieur en permanence) pour recevoir un implant. La majorité des chiennes étaient âgées de moins de trois ans, tandis que la majorité des mâles avaient moins de six ans. Les 513 chiens capturés en 2015 ont été vaccinés contre la rage et aucun cas de rage n'a été observé chez les chiens de la communauté depuis 2013.

\section{Figure 2 : Nombre de chiens capturés dans les communautés de l'autorité sanitaire de la région de Weeneebayko, 2015}

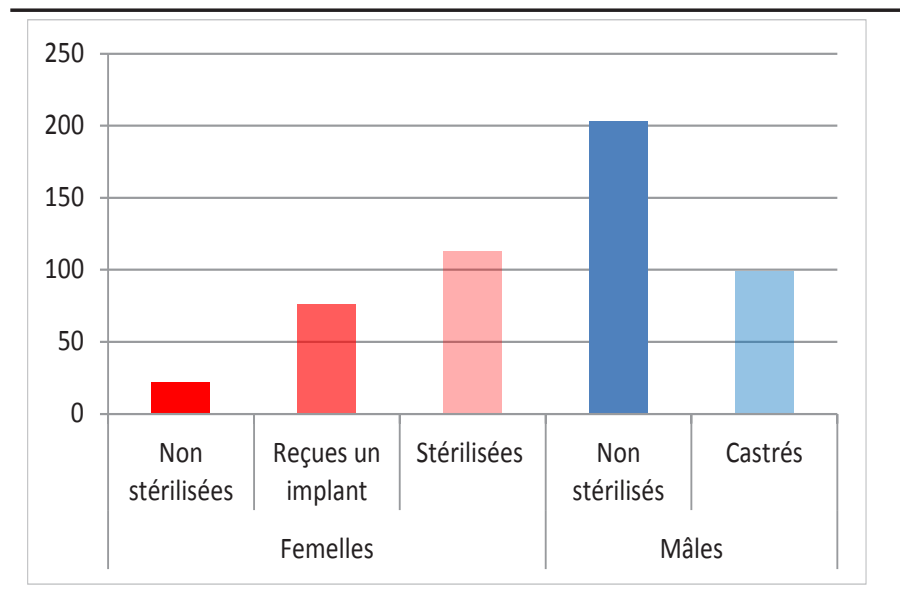


Pour chaque chien capturé, un registre du chien et un dossier médical ont été créés et associés au numéro de sa micropuce, notamment les renseignements sur le type de race, le sexe, l'âge, le statut de reproduction, la note d'état corporel, le poids approximatif, tout antécédent connu et les vaccins et les vermifuges administrés. Tous les renseignements recueillis sur les chiens ont été communiqués à chaque communauté en format électronique et en format écrit et l'autorité sanitaire de la région de Weeneebayko a conservé des copies de sauvegarde des données. Des cartes de densité des populations de chiens dans la communauté ont aussi été générées et indiquent les numéros de lot et les zones d'habitation des chiens, afin d'informer et de faciliter les interventions communautaires et dans le cadre du projet de l'autorité sanitaire de la région de Weeneebayko en 2016 et après. Chaque communauté a aussi reçu un lecteur de micropuce lui permettant de balayer, d'identifier et de déterminer le statut de vaccination antirabique de tous les chiens munis d'une micropuce, au besoin.

\section{Discussion}

Le projet de l'autorité sanitaire de la région de Weeneebayko a touché $60 \%$ de la population canine dans la région de Weeneebayko en 2015. Des vaccins antirabiques et DA2PP ont été administrés à tous les chiens et près de $80 \%$ des femelles non stérilisées ont reçu un contraceptif injectable efficace pendant 12 mois.

Les données recueillies dans le cadre du projet de l'autorité sanitaire de la région de Weeneebayko en 2015 ont révélé des différences importantes au sein des populations canines entre chaque communauté de la région, ce qui reflète leur degré d'accès aux services de stérilisation ou de castration avant 2015. Une majorité de chiens précédemment stérilisés (72\% des femelles stérilisées et $51 \%$ des mâles castrés) se trouvait dans les communautés les plus au Sud, c'est-à-dire celles qui avaient un meilleur accès aux cliniques de stérilisation ou de castration. Ces communautés ont aussi tendance à avoir un plus grand nombre de petits chiens d'intérieur (p. ex. Chihuahuas, Poméraniens, etc.) qui ne contribuent pas à la surpopulation canine et aux problèmes de sécurité publique. Cependant, il convient de noter que les communautés qui avaient certains animaux stérilisés grâce à un accès occasionnel à des services de stérilisation ou de castration (fournis uniquement de façon ponctuelle et sans coordination ni planification à long terme), n'avaient pas moins de problèmes de surpopulation canine que les communautés qui n'avaient jamais eu accès à de tels services. Cela semble dépendre d'un certain nombre de facteurs, notamment du fait que les cliniques de stérilisation ou de castration, en général, ne sortent pas de manière proactive pour capturer systématiquement tous les chiens errants, mais attendent plutôt que les membres de la communauté apportent les chiens à un endroit centralisé pour une intervention chirurgicale, et à la non-priorisation des interventions chirurgicales de stérilisation des femelles par rapport à la castration des mâles.

Ce point de vue a été adopté par une communauté de l'autorité sanitaire de la région de Weeneebayko qui constitue une exception notable dans la région. Cette communauté a déjà mis en œuvre une approche uniforme à l'égard des chiens errants sans propriétaire sur trois ans, notamment les cliniques de stérilisation ou de castration répétée visant les chiennes, la capture de chiens et le retrait des chiots des portées aux abords de la communauté à des fins d'adoption en dehors de la région. Toutes les chiennes de cette communauté ont été stérilisées et seule une poignée de mâles n'a pas été castrée. Des renseignements anecdotiques fournis initialement par les agents locaux des services policiers de la nation Nishnawbe-Aski dans cette communauté indiquaient une baisse importante du nombre d'appels liés à des chiens reçus par la police, provisoirement associés à la stérilisation réussie de la majorité des femelles dans la communauté.

Comme il a déjà été mentionné auparavant, les défis logistiques liés à l'entreprise d'un projet de régulation des populations canines dans une région éloignée ou isolée sont importants et nécessitent de nombreuses ressources pour se rendre dans cette région et expédier tout le matériel nécessaire. L'utilisation de contraceptifs injectables chez les chiennes comme mécanisme de stabilisation des populations réduit la quantité de matériel vétérinaire qu'il faut expédier au strict minimum, ce qui est un atout indiscutable dans le cadre de cette approche. En outre, les contraceptifs injectables ne nécessitent pas un suivi vétérinaire, comme c'est souvent le cas pour les interventions chirurgicales de stérilisation ou de castration. Dans le cadre de consultations avec les communautés avant de choisir une approche pour le projet de l'autorité sanitaire de la région de Weeneebayko, l'équipe du projet a remarqué que le personnel médical dans la majorité des communautés émettait souvent des préoccupations à propos du suivi des cliniques de stérilisation ou de castration et que l'on demande souvent aux postes de soins infirmiers communautaires de traiter les complications postopératoires vétérinaires, telles que les infections du site opératoire, pour lesquelles ils ne sont pas formés ou ne disposent pas du matériel nécessaire. Cela contribue aussi à la réticence de certains propriétaires de chiens à faire opérer leurs chiens.

Bien que la nature provisoire des contraceptifs injectables puisse être perçue comme une faiblesse dans le cadre de cette approche, les auteurs soutiendraient que cela serait le cas uniquement en cas d'intervention unique et non pas dans le cas d'un plan coordonné sur plusieurs années, comme c'est le cas pour le projet de l'autorité sanitaire de la région de Weeneebayko. L'administration d'implants aux chiennes pendant deux années consécutives assure leur infertilité jusqu'à trois ans, ce qui couvre la majorité de la vie des chiennes non stérilisées dans la région. Les chiennes qui survivent plus de trois ans dans le Nord sont de meilleures candidates pour des interventions de stérilisation chirurgicale. À la suite de la deuxième année d'utilisation d'un implant contraceptif dans la région en 2016, les prochaines étapes du projet de l'autorité sanitaire de la région de Weeneebayko incluront une analyse de l'ensemble des données de la dynamique des populations canines pendant ce projet de deux ans. Les données définitives du projet permettront d'envisager des options rentables de possible plan de stérilisation ou de castration chirurgicale coordonnée ou d'utilisation continue d'un implant contraceptif chez les chiennes dans les communautés de l'autorité sanitaire de la région de Weeneebayko à l'avenir.

Les conclusions de références de 2015 du projet de l'autorité sanitaire de la région de Weeneebayko soutiennent la faisabilité de l'utilisation d'implants contraceptifs en tant qu'intervention principale novatrice destinée à empêcher les cycles de reproduction des chiennes dans les régions éloignées du Nord 
en l'absence d'un accès régulier à des services vétérinaires. La reproduction continue en arrière-plan assurera une croissance continue de la population à long terme, malgré les efforts de stérilisation ou de castration, sauf si la majorité des chiennes de la communauté peut être stérilisée en une seule fois. Bien que nous soyons dans l'attente des résultats définitifs, les données recueillies dans le cadre du projet en 2015 indiquaient aussi que les animaux stérilisés ou castrés ne demeurent pas dans la communauté longtemps, sauf si la population générale est sous contrôle. Ajouté à cela les limites de temps ou de coût associées à la mise à la disposition de la stérilisation ou de castration dans la région, les conditions météorologiques extrêmes et le manque d'accès à des soins vétérinaires pour traiter les complications postopératoires telles que les infections du site opératoire, et tout vient appuyer l'utilisation d'implants contraceptifs en tant que meilleure approche que la stérilisation ou la castration chirurgicale dans la région comme méthode d'intervention principale. L'utilisation des contraceptifs doit être suivie par des approches de stérilisation ou de castration chirurgicale dans un délai de deux à trois ans, une fois que la croissance du reste de la population a fortement diminué, ou s'est arrêtée.

\section{Remerciements}

Les auteurs remercient la $D^{\text {re }}$ Catherine Filejski du ministère de la Santé et des Soins de longue durée de l'Ontario qui a fait preuve de leadership continu, qui a donné des conseils et a soutenu le projet, et qui a formulé des commentaires pertinents sur l'article, et la $D^{\text {re }}$ Judith Samson-French pour son leadership inestimable auprès de l'équipe sur le terrain et son expertise sur l'utilisation et l'administration d'implants contraceptifs chez la population canine.

\section{Financement}

Le financement des aspects vétérinaires du projet pilote de gestion de la population canine de l'autorité sanitaire de la région de Weeneebayko a été fourni généreusement par PetSmart Charities of Canada.

\section{Conflit d'intérêts}

Aucun.

\section{Références}

1. Garde E, Perez GE, Vanderstichel R, Dalla Villa PF, Serepell JA. Effects of surgical and chemical sterilization on the behavior of free-roaming male dogs in Puerto Natales, Chile. Prev Vet Med 2016 Jan 1;123:106-20.

2. Totton SC, Wandeler Al, Zinsstag J, Bauch CT, Ribble CS, Rosatte RC, McEwen SA. Stray dog population demographics in Jodhpur, India following a population control/rabies vaccination program. Prev Vet Med 2010 Oct 1;97(1):51-7.

3. Childs JE, Robinson LE, Sadek R, Madden A, Miranda ME, Miranda NL. Density estimates of rural dog populations and an assessment of marking methods during a rabies vaccination campaign in the Philippines. Prev Vet Med 1998 Jan;33(1-4):207-18.

4. Paul M, Sen Majumber S, Sau S, Nandi AK, Bhadra A. High early life mortality in free-ranging dogs is largely influenced by humans. Sci Rep 2016 Jan 25;6:19641.

5. Alliance for Contraception in Cats and Dogs (ACC\&D).

Contraception and fertility control in cats and dogs: A report of the Alliance for Contraception in Cats and Dogs. Portland OR: ACC\&D; 2013. https://www.acc-d.org/docs/default-source/Resource-LibraryDocs/accd-e-book.pdf?sfvrsn=0.

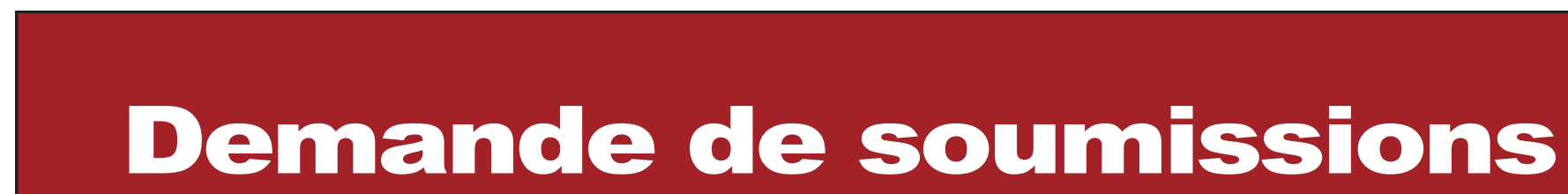

\section{Promotion de la santé et prévention des maladies chroniques au Canada}

Les rédacteurs de Promotion de la santé et prévention des maladies chroniques au Canada sont heureux d'annoncer que la revue invite à nouveau la soumission d'articles de tous les auteurs, indépendamment de leur rattachement.

Pour plus d'information et pour soumettre un article, veuillez consulter: phac-aspc.gc.ca/publicat/hpcdp-pspmc/autinfo-fra.php\#subman

Recherche, politiques et pratiques 